\title{
MicroRNA-Based Biomarkers in the Diagnosis and Monitoring of Therapeutic Response in Patients with Depression
}

This article was published in the following Dove Press journal: Neuropsychiatric Disease and Treatment

\author{
Ya-Yun $X u^{1-4}$ \\ Qian-Hui $X_{i a}{ }^{5}$ \\ Qing-Rong $X_{i a}{ }^{1,3,4}$ \\ Xu-Lai Zhang 6,7 \\ Jun Liang ${ }^{1,3,4}$
}

'Department of Pharmacy, Hefei Fourth People's Hospital, Hefei 230000, People's Republic of China; ${ }^{2}$ CAS Key Laboratory of Brain Function and Disease, University of Science and Technology of China, Hefei 230022, People's Republic of China; ${ }^{3}$ Department of Psychopharmacology, Anhui Mental Health Center, Hefei 230000, People's Republic of China; ${ }^{4}$ Department of Clinical Pharmacy, Affiliated Psychological Hospital of Anhui Medical University, Hefei 230000, People's Republic of China; ${ }^{5}$ School of Pharmacy, Wannan Medical College, Wuhu 241002, People's Republic of China; ${ }^{6}$ Department of Geriatric Psychology, Hefei Fourth People's Hospital, Hefei 230000, People's Republic of China; ${ }^{7}$ Clinical School of Mental Health, Anhui Medical University, Hefei 230000, People's Republic of China
Correspondence: Jun Liang Department of Pharmacy, Hefei Fourth People's Hospital, 316 Huangshan Road, Hefei 230000, People's Republic of China $\mathrm{Tel} / \mathrm{Fax}+8655163616022$

Email amhcliangj@।63.com

Xu-Lai Zhang

Department of Geriatric Psychology,

Hefei Fourth People's Hospital, 316

Huangshan Road, Hefei 230000, People's

Republic of China

$\mathrm{Tel} / \mathrm{Fax}+8655163616022$

Email Xulaizhang@ahmhcentre.com

\begin{abstract}
Depression is a debilitating mental illness that affects up to 120 million people worldwide; it is currently determined based on subjective diagnostic schemes that are limited by high uncertainty. Hence, there is an urgent need to identify effective and reliable biomarkers to increase diagnostic accuracy. MicroRNAs (miRNAs) constitute a recently discovered class of non-coding RNAs that play a key role in the regulation of gene expression by modulating translation, messenger RNA (mRNA) degradation, or stability of mRNA targets. Dysregulated expression of miRNAs is being investigated as a clinical biomarker for a variety of diseases, including depression. Accumulating evidence has shown that miRNAs participate in many aspects of neural plasticity, neurogenesis, and the stress response. This is supported by more direct studies based on human postmortem brain tissue that strongly indicate that miRNAs not only play a key role in the pathogenesis of major depressive disorder, but also present potential for the development of therapeutic targets. miRNAs in the peripheral and central nervous system are being considered as potential biomarkers in the diagnosis of depression and in monitoring the therapeutic response to antidepressants, owing to their stability, tissue-specificity, and disease-specific expression. In this review, we focus on various miRNAs in tissues and fluids that could be employed as diagnostic and therapeutic biomarkers in patients with depression.
\end{abstract}

Keywords: depression, microRNA, biomarker, diagnosis, therapy

\section{Introduction}

Depression is a debilitating mental illness and one of the main causes of suicide. It also presents an economic burden, with a high disability rate, that affects up to 120 million people worldwide (WHO 2017). ${ }^{1}$ The current diagnosis of depression is based primarily on a patient's self-reported experiences, behavior reported by relatives or friends, and a mental status exam; therefore, the uncertainty is as high as $30 \%{ }^{2}$ There is an urgent, unmet need to develop valid, reliable biomarkers to improve the diagnostic accuracy for depression. ${ }^{3}$ The current promising biomarkers of depression are neurotrophic factors, hypothalamic-pituitary-adrenal (HPA) axis-related hormones, inflammatory factors, and metabolism indexes; however, none of these are clinically useful owing to their limited sensitivity and specificity. ${ }^{4}$

MicroRNAs (miRNAs) are a class of short, non-coding RNAs, ranging from 17 to 25 nucleotides in length that bind to incomplete complementary regions in the $3^{\prime}$ untranslated region of target messenger RNAs (mRNAs), inhibiting their translation or causing their degradation. ${ }^{5}$ Numerous studies suggest that miRNAs are involved 
in a variety of biological processes associated with the central nervous system, including neurogenesis, neuronal proliferation, and synaptic plasticity. ${ }^{6,7}$ miRNAs in tissues and body fluids have several particularly attractive properties for laboratory science: they are stably maintained in a variety of biological fluids, including human serum/ plasma, urine, and cerebrospinal fluid; their separation is non-invasive; and their amplification is technically facile and inexpensive. ${ }^{8,9}$ Owing to their tissue-specific and disease-specific expression, stability, and repeatability, miRNAs have potential as biomarkers for illnesses and for predicting responses to different therapeutic strategies. $^{10,11}$ Accumulating evidence shows that dysregulation of miRNAs in bodily fluids and brain tissues may be associated with the depressed condition in both patients and animal models. ${ }^{12,13}$ Basal microRNA expression has also recently been shown to be predictive of the therapeutic response to antidepressants, consistent with their putative role as an antidepressant. ${ }^{14}$ These findings suggest that miRNAs in tissues and fluids can be used as biomarkers for diagnosis and monitoring therapeutic responses in patients with depression.

\section{Materials and Methods}

All included studies were retrieved from three databases: Web of Science, Scopus, and PubMed. The following keywords: "depression" or "depressive disorder" or "depressive behavior" or "depression-like behavior", and "microRNA" or "miRNA", and "diagnosis" or "therapy" or "antidepressant", and "biomarker" were used to search topics, keywords, and titles for relevant literature. Only original articles in English were included. The literature search was conducted between September 30, 2019, and October 30, 2019.

\section{miRNAs as Potential Biomarkers in the Diagnosis of Depression}

In this section, the involved participants were all either treatment-free, or their samples were analyzed for miRNA expression prior to the administration of antidepressants.

\section{miRNAs in Peripheral Blood Circulation}

The advantage of using blood-based markers, including serum/plasma and peripheral blood mononuclear cells (PBMCs), is the ease of collecting frequent samples from patients. Circulating miRNAs are also resistant to nuclease digestion and can be repeatedly measured, making them attractive as potential biomarkers. ${ }^{15}$

Many research groups are engaged in analyzing the aberrant expression of single serum/plasma miRNA in patients with depression and in controls. For example, it has been shown that plasma miR-144-5p levels at baseline in patients with depression were significantly lower compared with those in the healthy controls. ${ }^{16}$ The levels of miR-135, which is a prominent regulator of key 5-hydroxytryptamine (5-HT) system transcripts, is significantly lower in the blood of human patients with depression. ${ }^{17}$ Similarly, a decrease of miR-1202 levels in the blood of major depressive disorder (MDD) subjects has been reported. ${ }^{18}$ In contrast with the above down-regulated miRNA levels, several studies have found up-regulated miRNA levels in patients with depression. It has been reported that miR-124 may serve as a biomarker for depression because its level is increased in the plasma of drug-free patients with depression. ${ }^{19}$ miR-124-3p is derived from pre-miR-124-3, whose encoding gene is located on chromosome 3. Similar upregulation of miR124-3p was observed in blood serum of antidepressantfree MDD subjects. ${ }^{20}$ Combining the detection of different biomarkers is reported to be a useful strategy because of the increased sensitivity and specificity of each biomarker. Kuang et al determined that serum levels of miRNA-451a, miRNA-34a-5p, and miRNA-221-3p serve as indicators of disease progression in depression. ${ }^{21}$ Real-time quantitative reverse transcription polymerase chain reaction (qRTPCR) results showed that miRNA-451a expression levels were significantly decreased, while miRNA-34a-5p and miRNA-221-3p expression levels were significantly increased in patients with depression compared with those in the controls. ${ }^{21}$ Decreased brain-derived neurotropic factor (BDNF) protein levels have been suggested to affect the pathology of MDD, and Li et al have identified and characterized the roles of BDNF and its putative regulatory miRNAs in depression. ${ }^{22}$ The results showed that, compared with healthy controls, patients with depression showed lower serum BDNF levels and higher serum miR-132 and miR-182 levels. Furthermore, a reverse relationship between serum BDNF levels and miR-132/miR182 level was also found, indicating that the serum BDNF and its related miRNAs may be utilized as important biomarkers in the diagnosis of depression. ${ }^{22}$ Camkurt et al investigated miRNA dysregulation in plasma samples of drug-naive, first-episode patients with depression. ${ }^{23}$ They found that miR-320a levels were significantly 
downregulated and those of miR-451a, miR-17-5, and miR-223-3p were significantly upregulated in these patients. $^{23}$ Another study also selected drug-naive, firstepisode patients; the results showed that the expression levels of plasma miR-34b-5p and miR-34c-5p in MDD patients were significantly higher than those in control subjects. ${ }^{24}$ An additional study has confirmed that the expression level of plasma miR-34b-5p was significantly lower in patients with suicidal ideation, indicating that miR-34b-5p may be utilized as an important biomarker in the diagnosis of suicidal thoughts in patients with depression. $^{24}$

Multiple studies have shown that transcriptional changes in PBMCs may reflect alterations in molecules and cells in the brain. ${ }^{25,26}$ The central nervous system (CNS) may affect the gene expression of peripheral lymphocytes through cytokines, neurotransmitters, or hormones, which may explain the comparable gene expression levels between peripheral blood and some CNS tissues. ${ }^{27,28}$ Moreover, some evidence shows that PBMCs and brain tissue may share the same pattern of miRNA expression. ${ }^{29}$ Additionally, recent studies have shown that the abnormal expression of miRNAs in PBMCs may be related to the pathogenesis of MDD. ${ }^{30}$ Based on the evidence described above, several studies have explored the value of miRNA in PBMCs in the diagnosis of depression. It has been shown that expression levels of miR-124 from PBMCs in MDD patients were significantly higher than those in healthy controls. ${ }^{31}$ Moreover, qRT-PCR analysis in a large cohort of 81 MDD patients and 46 healthy controls confirmed that the expression levels of five miRNAs (miRNA-26b, miRNA1972, miRNA-4485, miRNA-4498, and miRNA-4743) were up-regulated. ${ }^{32}$ Furthermore, Belzeaux et al compared miRNA expression in PBMCs in 16 severe MDD patients and 13 matched controls and found significantly up- and also downregulated miRNAs (upregulated: miR-107, miR-133a, miR-148a, miR-425-3p, miR-494, miR-579, miR-589, miR-652, miR-941; downregulated: miR-200c, miR-381, miR-517b, miR-636, miR-1243). ${ }^{30}$

To evaluate the diagnostic ability of circulating miRNAs in depression, several studies conducted receiver operating characteristic (ROC) analysis to estimate the sensitivity and specificity. It has been shown that the area under the curve of let-7d-3p, miR-34a-5p, miR-221-3p, and miR-451a in serum was 0.94, 0.98, 0.97, and 0.94, with specificities of $90.48 \%, 95.24 \%, 90.48 \%$, and $90.48 \%$, and sensitivities of $93.75 \%, 96.88 \%, 90.63 \%$, and $84.85 \%$, respectively, in distinguishing MDD patients from healthy controls. ${ }^{33}$ Moreover, the area under the curve of miR-124 in PBMCs from ROC analysis was 0.762 with a sensitivity of $83.33 \%$ and specificity of $66.67 \%$ in distinguishing MDD patients from healthy controls. $^{31}$ The combination of multiple miRNAs has proven to be a more comprehensive and effective indicator for disease detection than single miRNA; ${ }^{34,35}$ accordingly, Fan et al examined the predictive power of five miRNAs (miRNA-26b, miRNA-1972, miRNA-4485, miRNA-4498, and miRNA-4743) in PBMCs as a holistic panel for MDD diagnosis. $^{32}$ The results showed the combined area under the ROC curve was 0.6361 , with $54.35 \%$ sensitivity and $79.01 \%$ specificity. ${ }^{32}$ These results suggest that the changes in circulating miRNA expression can be used as biomarkers for the diagnosis of depression.

\section{miRNAs in Dermal Fibroblasts}

Dermal fibroblasts are easily established and maintained in culture without any transformation, and after several rounds of cell division, most confounding factors (such as lifestyle or drug use) are virtually eliminated. ${ }^{36}$ Previous studies support the notion that human fibroblasts represent a relevant model for studying processes of signal transduction in patients with affective disorders because they have many similarities with neuronal tissue in signal transduction. ${ }^{37,38}$ Moreover, a recent study supported the role of oxidative stress in the pathophysiology of MDD by assaying human dermal fibroblast cultures derived from skin biopsies of individuals in depressed and normal control groups. ${ }^{39}$ More recently, a combined mRNA-miRNA profiling of dermal fibroblasts from patients with MDD and matched controls was performed to explore promising peripheral biomarkers of MDD. ${ }^{36}$ The data revealed that, compared with matched controls, the MDD patient fibroblasts showed an altered expression of a distinct panel of 38 miRNAs, indicating that analyzing changes in miRNA expression in dermal fibroblasts might lead to the discovery of promising peripheral biomarkers for MDD. ${ }^{36}$

\section{MiRNAs in Cerebrospinal Fluid (CSF) Examination}

It has been reported that alterations in CSF miRNAs can reflect the changes of miRNAs in brain pathological tissue better than those in blood or postmortem brain samples. ${ }^{40}$ There are two reasons for this phenomenon: ${ }^{41,42}$ (1) In view of the inherent limitations associated with obtaining 
brain tissue in vivo, most human studies use postmortem samples to study miRNA expression. However, the study of postmortem brain tissue may be confounded by an extended postmortem interval and prior medication exposure; (2) Peripheral blood may not closely reflect gene expression levels in the brain. Considering the intimate relationship between the brain and $\mathrm{CSF}$, the application of CSF-derived indicators represents a new method for identifying central nervous system (CNS) biomarkers. Wan et al detected the differential miRNAs in the CSF of MDD patients. ${ }^{33}$ The results indicated that 16 miRNAs in CSF were differentially expressed between the control group and patients with depression. ${ }^{33}$ Specifically, the mean miR-125a-5p, let-7d-3p, miR-30a-5p, miR-34a-5p, miR-221-3p, miR-29b-3p, miR-10a-5p, miR-375, miR155-5p, miR-33a-5p, and miR-139-5p levels in the CSF of patients with depression were notably higher than those in control patients, while the mean miR-451a, miR-15b-5p miR-106b-5p, miR-590-5p, and miR-185-5p levels in the CSF of patients with depression were notably lower than those in control patients. ${ }^{33}$ Song et al recruited 36 drugfree patients with MDD and 30 healthy controls to investigate the possible involvement of CSF miR-16 in the mechanism of MDD in humans. ${ }^{43}$ The results showed that CSF miR-16 in patients with MDD was significantly lower than that in the controls. ${ }^{43}$ Moreover, correlation analysis indicated that CSF miR-16 levels were negatively correlated with Hamilton scores and positively associated with CSF serotonin levels, ${ }^{43}$ suggesting that CSF miR-16 participated in the physiopathology of MDD via modulation of the serotonin transmitter system in the brain.

\section{MiRNAs in Brain Tissues}

The hippocampus is the area of the brain most frequently studied in research into depression. ${ }^{44,45}$ From a structural point of view, the hippocampus is part of the limbic system and develops nerve fiber connectivity with emotion-related brain regions, for instance, the prefrontal cortex and amygdala. ${ }^{46}$ In addition, the hippocampus contains high levels of glucocorticoid receptors and glutamate and regulates the HPA axis, ${ }^{47}$ which makes it more susceptible to stress and depression. ${ }^{48}$ Previous studies have demonstrated that hippocampal plasticity in depression involves hippocampal volumetric changes, neurogenesis, and apoptosis of neurons. ${ }^{49,50}$ Recently, the expression of several miRNAs has been found to be altered in the hippocampus in animal models of depression. Preclinical studies have investigated changes to hippocampal miRNA levels induced via the maternal separation (MS) procedure, a well validated stress model, in Sprague-Dawley rats. ${ }^{51}$ By employing a microarray, it has been found that MS significantly alters the expression levels of 24 different miRNAs in the hippocampus, of which the two of most interest (downregulated: miR-451; upregulated: miR-598$5 \mathrm{p})$ have also been validated by qRT-PCR. ${ }^{51}$ In another preclinical study, chronic unpredictable mild stress (CUMS), a well-known effective model that imitates the pathogenesis of depression, has been chosen to explore critical roles in this process. ${ }^{14}$ The results showed that the expression of miR-132 and miR-18a in the hippocampus in the model group were significantly lower than in the control group, whereas the level of miR-134 and miR$124 \mathrm{a}$ were significantly higher in the model group than in the control group. ${ }^{14}$ These alterations were reversed by treatment with duloxetine, suggesting that the hippocampus may represent a site of action of duloxetine, whose mechanism of action may partially involve the regulation of miRNA levels. ${ }^{14}$

Although the underlying mechanism of depression is not clear, accumulating evidence suggests that stress and subsequent HPA axis hyperactivity in the hypothalamus may be the most important etiological risk factor for the onset of depression. ${ }^{52}$ Given the fact that the hypothalamus plays a crucial role in integrating signals from other brain nuclei as well as environmental, hormonal, metabolic, and neuronal signals from the periphery in order to deliver an adequate response, it is rational to hypothesize that hypothalamic miRNA levels may become dysregulated during depression. However, to the best of our knowledge, only one study has focused on the altered status of hypothalamic miRNAs in an animal depression model. ${ }^{53}$ The results showed that, compared with the sham group, the miR-99a level decreased significantly in the hypothalamus during "postmenopausal depression" by using a novel "peri- to postmenopausal depression" model induced by a two-step ovariectomy plus chronic mild stress (CMS). ${ }^{53}$

Structural and functional imaging studies have shown that the prefrontal cortex (PFC) plays an important role in the regulation of emotion and is related to some neurobiological abnormalities involved in the pathophysiology of depression and suicide. ${ }^{54,55}$ Several human studies have examined whether the miRNA network is altered in the PFC of depressed suicide subjects. In the study of Smalheiser et al, expression of miRNAs was measured in the PFC of antidepressant-free depressed suicides $(\mathrm{n}=18)$ 
and well-matched non-psychiatric control subjects $(\mathrm{n}=17)$ using multiplex RT-PCR plates. ${ }^{56}$ The results showed that 21 miRNAs including miR-101, miR-137, miR-142-5p, miR-146a, miR-489, and miR-494 were significantly decreased in the suicidal group. ${ }^{56}$ Many of the downregulated miRNAs were encoded at nearby chromosomal loci, shared motifs within the 59-seeds, and shared putative mRNA targets, several of which have been implicated in depression. ${ }^{56}$ Moreover, in a microarray screening study, miR-185 and miR-491-3p were upregulated in the PFC of suicides. ${ }^{57}$ Furthermore, Lopez et al found that several miRNAs (miR-34c-5p, miR-139-5p, miR-195, and miR-320c) showed significant up-regulation in the PFC of suicides who were all with a history of major depressive disorder based on DSM-IV criteria compared with that in psychiatric healthy controls. ${ }^{58}$ In addition, another study revealed that the expression of miR-30a and miR-30e was significantly increased, while the expression of miR-200a was drastically reduced in samples from individuals with MDD who died by suicide. ${ }^{59}$ These studies suggest a relationship between altered miRNAs in the PFC and suicidal behavior in subjects with depression. Consistent with the clinical studies, animal studies have also demonstrated dysregulated miRNA expression in the PFC of animal depression models. Chronic corticosteroneinduced depression in rodents is a well-established animal model that allows examination of the direct effects of glucocorticoids on the development of depressive symptomatology. ${ }^{60}$ It has been reported that chronic corticosterone administration to rats causes altered expression of 26 miRNAs in the PFC. ${ }^{61}$ Among these, 19 were upregulated (let-7i, miR-19b, miR-29c, miR-101a, miR124, miR-137, miR-153, miR-181a, miR-181c, miR-203, miR-218, miR-324-5p, miR-365, miR-409-5p, miR-5825p, miR-155, miR-29a, miR-30e, miR-721, miR-699) and seven were downregulated (miR-146a, miR-200c, miR351, miR-155, miR-678, miR-764-5p, miR-135a*). ${ }^{61}$ These changes in miRNA expression levels in the PFC reflect the responsiveness to a hyperactive HPA axis as a direct representation of increased serum corticosterone level in corticosterone-treated rats that may be related to the development of a depressive phenotype. CUMS, another widely used depression model closely related to the HPA axis, ${ }^{62}$ has been used to explore the role of miRNAs in the PFC during depression. ${ }^{63}$ The results showed that increasing the duration of CUMS increased miR-124 levels in murine PFCs. Furthermore, miR-124 overexpression, by using lentiviral transfection in murine
PFCs that received CUMS, exacerbated depression-like behaviors, indicating that manipulation of miR-124 can greatly alter depression-like behaviors induced by CUMS. ${ }^{63}$ Additionally, chronic pain and depression are often accompanied by common clinical manifestations and biological connections related to sensitization of the CNS. ${ }^{64}$ In a comorbidity animal model of depression and chronic pain that was subjected to CUMS and spared nerve injury (SNI) to initiate depressive-like behavior and chronic pain behavior, respectively, five specific miRNAs (miR-10a-5p, miR-182, miR-200a-3p, miR200b-3p, and miR-429) in the PFC were simultaneously down-regulated after four weeks of stress. ${ }^{65}$ Among these, miR-200a-3p expression was significantly up-regulated after 12 weeks of stress, ${ }^{65}$ suggesting that miR-200a-3p might be a specific biomarker of central sensitization in chronic pain and depression. The main miRNAs isolated from the PBMCs, serum or plasma, fibroblasts, hippocampus, PFC, and hypothalamus, that play a potential role in the diagnosis of depression, are shown in Table 1. These reports indicate the importance of miRNAs as potential biomarkers for the diagnosis of depression.

\section{miRNA Modulation in Response to Antidepressant Treatment}

Monitoring the response of patients with depression using appropriate biomarkers is another important aspect of treatment. $^{73}$ A variety of evidence suggests that several antidepressants or therapeutic methods, including the administration of selective serotonin reuptake inhibitors (SSRIs), serotonin and norepinephrine reuptake inhibitor (SNRIs), or ketamine and electroconvulsive therapy (ECT), exert their effects by targeting miRNAs. This suggests that miRNAs may be used as powerful biomarkers for monitoring response to therapy in patients with depression. $^{74,75}$

\section{miRNA Modulation in Response to SSRIs}

SSRIs are the most commonly prescribed antidepressants owing to their ability to alleviate symptoms of moderate to severe depression; additionally, they are relatively safe and generally produce fewer side effects than other types of antidepressants. ${ }^{76}$ Currently, six SSRIs are commonly used in clinical practice: fluoxetine, paroxetine, sertraline, fluvoxamine, citalopram, and escitalopram.

The first study on the antidepressant effect of fluoxetine on miRNA alterations in animals showed that the 
Table I Selected miRNA Candidates Which are Correlated to Diagnosis in Depression

\begin{tabular}{|c|c|c|c|c|c|c|c|}
\hline MiRNA & PBMCs & Serum or Plasma & CSF & Fibroblast & Hippocampus & PFC & Hypothalamus \\
\hline miR-let-7i & & & & & & $\uparrow[6 \mid]$ & \\
\hline miR-7 & & & & $\downarrow[36]$ & & & \\
\hline miR-7a & & & & & $\uparrow[66]$ & & \\
\hline miR-7b & & $\downarrow[67]$ & & & & & \\
\hline miR-7d-3p & & $\uparrow[33]$ & $\uparrow[33]$ & & & & \\
\hline miR-I0a & & & & & & $\downarrow[56]$ & \\
\hline miR-10a-5p & & & $\uparrow[33]$ & & & $\downarrow[65]$ & \\
\hline miR-I5b & & & & & $\downarrow[66]$ & & \\
\hline miR-15b-5p & & & $\downarrow[33]$ & & & & \\
\hline miR-16 & & $\downarrow[68]$ & $\downarrow[43]$ & & $\uparrow[69]$ & & \\
\hline miR-16-2 & & & & $\downarrow[36]$ & & & \\
\hline miR-I7-5p & & $\uparrow[23]$ & & & & & \\
\hline $\operatorname{miR}-18 a$ & & & & & $\downarrow[14]$ & & \\
\hline miR-19b & & & & & & $\uparrow[6 \mid]$ & \\
\hline miR-20a & & & & & & $\downarrow[56]$ & \\
\hline $\mathrm{miR}-20 \mathrm{~b}$ & & & & & & $\downarrow[56]$ & \\
\hline miR-2I & & & & $\uparrow[36]$ & & & \\
\hline $\mathrm{miR}-24-3 \mathrm{P}$ & & & & & & $\uparrow[70]$ & \\
\hline miR-26b & $\uparrow[32]$ & & & & & & \\
\hline $\mathrm{miR}-27 \mathrm{a}$ & & & & & & $\downarrow[56]$ & \\
\hline miR-29a & & & & & & $\uparrow[6 \mid]$ & \\
\hline miR-29b-3p & & & $\uparrow[33]$ & & & & \\
\hline $\mathrm{miR}-29 \mathrm{c}$ & & & & & & $\uparrow[6 \mid]$ & \\
\hline miR-30a & & & & & & $\uparrow[59]$ & \\
\hline miR-30a-5p & & & $\uparrow[33]$ & & & & \\
\hline miR-30e & & $\uparrow[7 I]$ & & & & $\uparrow[59,61]$ & \\
\hline miR-32 & & & & $\uparrow[36]$ & & & \\
\hline miR-33a & & & & $\downarrow[36]$ & & & \\
\hline miR-33a-5p & & & $\uparrow[33]$ & & & & \\
\hline miR-34a-5p & & $\uparrow[2 \mathrm{I}, 33]$ & $\uparrow[33]$ & & & & \\
\hline miR-99a & & & & & & & $\downarrow[53]$ \\
\hline miR-I0I & & & & & & $\downarrow[56]$ & \\
\hline miR-IOIa & & & & & & $\uparrow[6 I]$ & \\
\hline miR-I03a & & & & $\uparrow[36]$ & & & \\
\hline miR-106b-5p & & & $\downarrow[33]$ & & & & \\
\hline miR-I07 & $\uparrow[30]$ & & & $\uparrow[36]$ & & & \\
\hline miR-122 & & & & $\uparrow[36]$ & & & \\
\hline miR-I24 & $\uparrow[31]$ & $\uparrow[19,72]$ & & & $\uparrow[66]$ & $\uparrow[61,63]$ & \\
\hline miR-I24a & & & & & $\uparrow[14]$ & & \\
\hline miR-124-3p & & $\uparrow[20]$ & & & & & \\
\hline miR-125a & & & & & $\downarrow[66]$ & & \\
\hline miR-125a-5p & & & $\uparrow[33]$ & & & & \\
\hline miR-130a & & & & & & $\downarrow[56]$ & \\
\hline miR-I30b & & & & & $\downarrow[66]$ & & \\
\hline miR-I32 & & $\uparrow[22,7 \mathrm{I}, 72]$ & & $\downarrow[36]$ & $\downarrow[14]$ & & \\
\hline miR-I33a & $\uparrow[30]$ & & & & & & \\
\hline miR-I34 & & & & & $\uparrow[14]$ & & \\
\hline miR-I35 & & $\downarrow[17]$ & & & & & \\
\hline miR-I35a & & $\downarrow[68]$ & & & $\downarrow[66]$ & $\downarrow[6 I]$ & \\
\hline miR-I35b & & & & $\downarrow[36]$ & & & \\
\hline miR-I37 & & & & & & $\uparrow[6 I] \downarrow[56]$ & \\
\hline
\end{tabular}

(Continued) 
Table I (Continued).

\begin{tabular}{|c|c|c|c|c|c|c|c|}
\hline MiRNA & PBMCs & Serum or Plasma & CSF & Fibroblast & Hippocampus & PFC & Hypothalamus \\
\hline miR-139 & & & & & $\uparrow[66]$ & & \\
\hline miR-139-5p & & & $\uparrow[33]$ & & & & \\
\hline miR-140-5p & & $\uparrow[73]$ & & & & & \\
\hline miR-142-3p & & & & & & $\downarrow[56]$ & \\
\hline miR-142-5p & & & & & & $\downarrow[56]$ & \\
\hline miR-144-5p & & $\downarrow[16]$ & & & & & \\
\hline miR-146a & & & & & & $\downarrow[56,61]$ & \\
\hline miR-146a-5p & & & & & & $\uparrow[70]$ & \\
\hline miR-146b-5p & & & & & & $\uparrow[70]$ & \\
\hline miR-148a & $\uparrow[30]$ & & & & & & \\
\hline miR-I48b & & & & & & $\downarrow[56]$ & \\
\hline miR-I53 & & & & & & $\uparrow[6 I]$ & \\
\hline miR-I55 & & & & & & $\downarrow[56,6 I]$ & \\
\hline miR-155-5p & & & $\uparrow[33]$ & & & & \\
\hline miR-I8Ia & & & & & & $\uparrow[6 \mid]$ & \\
\hline $\mathrm{miR}-18 \mathrm{Ic}$ & & & & & & $\uparrow[6 \mid]$ & \\
\hline miR-I82 & & $\uparrow[22]$ & & & $\uparrow[66]$ & $\downarrow[65]$ & \\
\hline miR-I85 & & $\uparrow[7 I]$ & & $\uparrow[36]$ & & & \\
\hline miR-185-5p & & & $\downarrow[33]$ & & & & \\
\hline miR-186 & & & & $\uparrow[36]$ & & & \\
\hline miR-190 & & & & & & $\downarrow[56]$ & \\
\hline miR-193a-3p & & & & $\uparrow[36]$ & & & \\
\hline miR-196b & & & & $\uparrow[36]$ & & & \\
\hline miR-200a & & & & & & $\downarrow[59]$ & \\
\hline miR-200a-3p & & & & & & $\downarrow[65]$ & \\
\hline miR-200b-3p & & & & & & $\downarrow[65]$ & \\
\hline miR-200c & $\downarrow[30]$ & & & & & $\downarrow[6 \mid]$ & \\
\hline miR-203 & & & & & & $\uparrow[6 \mid]$ & \\
\hline miR-2I 2 & & $\uparrow[7 \mid]$ & & & $\uparrow[66]$ & & \\
\hline miR-2। 8 & & & & & & $\uparrow[61] \downarrow[74]$ & \\
\hline miR-22I-3p & & $\uparrow[21,33,73]$ & $\uparrow[33]$ & & & & \\
\hline miR-223-3p & & $\uparrow[23]$ & & & & & \\
\hline miR-296-5p & & & & $\uparrow[36]$ & & & \\
\hline miR-298 & & & & & $\downarrow[66]$ & & \\
\hline miR-30la & & & & & & $\downarrow[56]$ & \\
\hline miR-320a & & $\downarrow[23]$ & & & & & \\
\hline miR-323 & & & & & $\downarrow[66]$ & & \\
\hline miR-324-5p & & & & & & $\uparrow[56,61]$ & \\
\hline miR-335 & & & & & & $\downarrow[56]$ & \\
\hline miR-337-5p & & & & $\uparrow[36]$ & & & \\
\hline miR-35I & & & & & & $\downarrow[6 I]$ & \\
\hline miR-365 & & & & & & $\uparrow[6 \mathrm{I}]$ & \\
\hline miR-375 & & & $\uparrow[33]$ & & & & \\
\hline miR-376a* & & & & & & $\downarrow[56]$ & \\
\hline miR-377 & & & & $\uparrow[36]$ & & & \\
\hline miR-38I & $\downarrow[30]$ & & & & & & \\
\hline$m i R-409-5 p$ & & & & & & $\uparrow[6 \mid]$ & \\
\hline miR-42I & & & & $\downarrow[36]$ & & & \\
\hline miR-424 & & & & $\downarrow[36]$ & & & \\
\hline miR-425 & & & & $\uparrow[36]$ & & & \\
\hline
\end{tabular}

(Continued) 
Table I (Continued).

\begin{tabular}{|c|c|c|c|c|c|c|c|}
\hline MiRNA & PBMCs & Serum or Plasma & CSF & Fibroblast & Hippocampus & PFC & Hypothalamus \\
\hline miR-425-3p & $\uparrow[30]$ & & & & & $\uparrow[70]$ & \\
\hline miR-429 & & & & $\downarrow[36]$ & & $\downarrow[65]$ & \\
\hline$m i R-450 a$ & & & & $\downarrow[36]$ & & & \\
\hline miR-45I & & & & & $\downarrow[5 ।]$ & & \\
\hline $\mathrm{miR}-45 \mathrm{Ia}$ & & $\downarrow[2 \mathrm{I}, 33] \uparrow[23]$ & $\downarrow[33]$ & & & & \\
\hline miR-489 & & & & & & $\downarrow[56]$ & \\
\hline miR-494 & $\uparrow[30]$ & & & & & $\downarrow[56]$ & \\
\hline miR-497 & & & & & & $\downarrow[56]$ & \\
\hline miR-503 & & & & & $\downarrow[66]$ & & \\
\hline miR-5I7b & $\downarrow[30]$ & & & & & & \\
\hline miR-532 & & & & & $\downarrow[66]$ & & \\
\hline miR-542-3p & & & & $\downarrow[36]$ & & & \\
\hline miR-548aa & & & & $\downarrow[36]$ & & & \\
\hline$m i R-548 d-3 p$ & & & & $\downarrow[36]$ & & & \\
\hline miR-579 & $\uparrow[30]$ & & & & & & \\
\hline miR-582-5p & & & & & & $\uparrow[6 \mid]$ & \\
\hline miR-589 & $\uparrow[30]$ & & & & & & \\
\hline miR-590-5p & & & $\downarrow[33]$ & & & & \\
\hline miR-598-5p & & & & & $\uparrow[5 \mathrm{I}]$ & & \\
\hline miR-6I3 & & & & $\downarrow[36]$ & & & \\
\hline miR-628-3p & & & & $\downarrow[36]$ & & & \\
\hline miR-629 & & & & $\downarrow[36]$ & & & \\
\hline miR-636 & $\downarrow[30]$ & & & & & & \\
\hline miR-652 & $\uparrow[30]$ & & & & & & \\
\hline miR-660 & & & & & & $\downarrow[56]$ & \\
\hline miR-66I & & & & $\downarrow[36]$ & & & \\
\hline miR-675 & & & & $\uparrow[36]$ & & & \\
\hline miR-678 & & & & & & $\downarrow[6 \mid]$ & \\
\hline miR-699 & & & & & & $\uparrow[6 \mid]$ & \\
\hline miR-72I & & & & & & $\uparrow[6 \mid]$ & \\
\hline miR-764-5p & & & & & & $\downarrow[6 \mid]$ & \\
\hline miR-887 & & & & $\uparrow[36]$ & & & \\
\hline miR-94I & $\uparrow[30]$ & & & & & & \\
\hline miR-I 202 & & $\downarrow[18,68]$ & & & & & \\
\hline miR-I243 & $\downarrow[30]$ & & & & & & \\
\hline miR-I246 & & $\downarrow[73]$ & & & & & \\
\hline miR-I 294 & & & & $\downarrow[36]$ & & & \\
\hline miR-197I & & & & & & $\downarrow[75]$ & \\
\hline miR-I972 & $\uparrow[32]$ & & & & & & \\
\hline miR-3I76 & & & & $\uparrow[36]$ & & & \\
\hline miR-37I4 & & & & $\downarrow[36]$ & & & \\
\hline miR-3909 & & & & $\downarrow[36]$ & & & \\
\hline $\operatorname{miR}-4267$ & & & & $\downarrow[36]$ & & & \\
\hline miR-4293 & & & & $\downarrow[36]$ & & & \\
\hline miR-4485 & $\uparrow[32]$ & & & & & & \\
\hline miR-4498 & $\uparrow[32]$ & & & & & & \\
\hline $\mathrm{miR}-4743$ & $\uparrow[32]$ & & & & & & \\
\hline
\end{tabular}

Notes: $\uparrow:$ upregulated, $\downarrow$ : downregulated; *When relative expression levels are known, an asterisk following the name indicates an miRNA expressed at low levels relative to the miRNA in the opposite arm of a hairpin. 
expression of miR-16 in the raphe nucleus of mice increased significantly after fluoxetine treatment. ${ }^{77}$ Further animal studies showed that decreased hippocampal miRNA levels (miR-451 and miR-598-5p), induced by the MS procedure, were reversed by fluoxetine treatment. ${ }^{51}$ The first in vitro evidence revealed that three (miR-489, miR-572, and miR-663a) and four (miR-320a, miR-489, miR-572, and miR-663a) miRNAs were up-regulated in two neuroblastoma cell lines (SK-N-SH cells and SHSY5Y cells), respectively, after a $24 \mathrm{~h}$ treatment with fluoxetine. $^{78}$

Lopez et al explored the regulation of miR-1202 in MDD patients receiving citalopram treatment. ${ }^{18}$ Based on changes in Hamilton Rating Scale for Depression (HAM-D) scores, depressed subjects were classified into remitters (REM) and non-responders (NRES). The REM group showed increased miR-1202 levels after eight weeks of citalopram treatment whereas there were no differences in the NRES or controls. ${ }^{18}$ Moreover, a change in depression severity was negatively correlated with change in miR-1202 expression, confirming a relationship between peripheral miR-1202 expression and citalopram treatment response in MDD patients. ${ }^{18}$ Another study enrolled 14 MDD patients who were prescribed citalopram and received treatment for two months. ${ }^{79}$ A decreasing trend in plasma miR-132 levels and a significant increase in plasma miR-124 levels after treatment were observed. ${ }^{79}$

Escitalopram, an SSRI and the S-enantiomer of the racemic compound citalopram, is used to restore serotonergic function in the treatment of depression and anxiety. ${ }^{80}$ Amongst SSRIs, escitalopram exerts the highest degree of selectivity for the serotonin transporter (SERT) relative to other off-targets, which may explain its lower rates of adverse effects compared with other agents in this class. ${ }^{81}$ Animal studies have reported that CUMS induces depression-like behavior and increases the expression of miR-326 in the nucleus accumbens, and decreases it in the striatum. ${ }^{82}$ Moreover, aberrant levels of miR-326 were shown to be normalized by a four-week treatment of escitalopram, suggesting that miR-326 in the nucleus accumbens and striatum plays a crucial role in the therapeutic effect of escitalopram. ${ }^{82}$ Clinical studies have shown that 30 miRNAs were differentially expressed after 12 weeks of treatment with escitalopram (28 miRNAs were up-regulated, and two miRNAs were strongly down-regulated) in the blood of 10 subjects with depression. ${ }^{83}$ miRNA target gene prediction and functional annotation analysis indicated significant enrichment in several pathways associated with neuronal brain function, supporting the hypothesis that the differentially regulated miRNAs may be involved in the antidepressant mechanism of escitalopram. ${ }^{83}$

Paroxetine is a novel phenylpiperidine antidepressant agent that acts as a potent and selective inhibitor of serotonin reuptake. ${ }^{84} \mathrm{An}$ in vitro study showed that paroxetine treatment rapidly increased brain-derived neurotrophic factor (BDNF) expression in the human glioblastoma-astrocytoma cell line U87. ${ }^{85}$ Simultaneously, the transcriptional BDNF inhibitor miR-30a-5p was also overexpressed. ${ }^{85}$ These findings indicate that the increased expression of BDNF induced by paroxetine may be related to the alteration of miR-30a-5p levels. The relationship between miRNAs and paroxetine has also been confirmed in another in vitro study, ${ }^{86}$ in which 80 human lymphoblastoid cell lines (LCLs) from healthy adult female individuals were screened for the induction of growth inhibition by paroxetine. The authors detected 6.71-fold higher basal expression levels of miR-151-3p in LCLs, with higher paroxetine sensitivity. ${ }^{86}$ Clinical studies have further confirmed the relationship between miRNAs and paroxetine. Kuang et al studied whether serum levels of miRNA-451a, miRNA-34a-5p, and miRNA-221-3p could serve as indicators of therapeutic efficacy in depression. ${ }^{21}$ They collected data from 84 patients with depression and found that paroxetine treatment was markedly effective in 50 patients, who also displayed an increased level of miRNA-451a but reduced levels of miRNA-34a-5p and miRNA-221-3p. ${ }^{21}$ Moreover, after antidepressant treatment, the miRNA-451a level was negatively correlated with the HAM-D score, while miRNA-34a-5p and miRNA-221-3p levels were positively correlated with HAM-D scores, ${ }^{21}$ suggesting that the expression levels of these miRNAs are closely related to the efficacy of paroxetine.

\section{miRNA Modulation in Response to SNRIs}

SNRIs have been proposed to exert an increased therapeutic effect or be faster acting compared with commonly used antidepressants because of their balanced effects on 5-HT and norepinephrine (NE) and their higher tolerability. ${ }^{87-89}$ Collective evidence has shown that miRNAs may be affected by SNRI treatment.

Duloxetine, an SNRI, has been used to treat MDD in clinical settings. ${ }^{90}$ Animal studies have shown that longterm treatment with duloxetine significantly ameliorated CUMS procedure-induced depression-like behavior. ${ }^{14}$ 
Moreover, duloxetine treatment has been found to induce a significant upregulation of miR-132 and miR-18a, and a significant downregulation of miR-134 and miR-124a, in the hippocampus of rats receiving CUMS. ${ }^{14}$ Furthermore, miR-18a showed significant upregulation in the frontal lobes of the duloxetine treatment group relative to the CUMS group. ${ }^{14}$ These findings suggest that the hippocampus and frontal lobe serve as the sites of action of duloxetine, whose mechanism of action presumably involves regulation of miRNA expression levels. A consistent alteration of miRNAs in the antidepressant response to duloxetine has also been observed in human studies. Lopez et al investigated miRNAs as biomarkers of an antidepressant response using small-RNA- sequencing in paired samples from MDD patients that were enrolled in a large, randomized placebo-controlled trial of duloxetine, collected before and eight weeks after treatment. ${ }^{91}$ After duloxetine treatment, they found significant downregulation in the expression of miR-146a-5p, miR-146b-5p, miR-425-3p, miR-24-3p, and miR-3074-5p in the blood of patients with depression. ${ }^{91}$ In addition, these miRNAs alterations were replicated in two independent clinical trials involving patients with depression who were treated with escitalopram (an SSRI) or nortriptyline (a tricyclic antidepressant), indicating that these miRNAs are consistent markers of antidepressant treatment. ${ }^{91}$

Desvenlafaxine, an active metabolite of venlafaxine, is another SNRI antidepressant drug. After eight weeks of desvenlafaxine treatment, peripheral miR-1202 levels were decreased in depressed patients. ${ }^{92}$ Furthermore, changes in peripheral miR-1202 levels were found to be associated with changes in brain activity and connectivity in a network of brain regions associated with depression and the antidepressant response, including ventral and dorsal prefrontal cortex, insula, temporal cortex, posterior cingulate, parietal (notably precuneus), occipital cortices, subcortical nuclei (notably the putamen), and claustrum. ${ }^{92}$ These findings suggest that miR-1202 levels modulate the antidepressant response to desvenlafaxine via effects on brain circuitry functioning, possibly via the glutamatergic system due to a trend for a significant correlation between miR-1202 levels and glutamate levels. ${ }^{92}$

\section{miRNA Modulation in Response to Other Drugs or Treatments for Depression}

Several studies have examined the miRNA alterations in patients with depression receiving individually tailored pharmacotherapy according to the currently accepted therapeutic guidelines of the American Psychiatric Association. It was reported that the expression levels of miR-124 were significantly down-regulated after eight weeks of treatment. ${ }^{31}$ Moreover, Belzeaux et al found that peripheral miR-20b-3p, miR-433, miR-409-3p, miR410, miR-485-3p, miR-133a, and miR-145 levels were upregulated and miR-331-5p levels were downregulated compared with the baseline levels in MDD patients after antidepressant treatment. ${ }^{30}$

Recently, ketamine, an N-Methyl-D-Aspartate (NMDA) receptor antagonist, has been shown to quickly and effectively reduce the symptoms of depression. ${ }^{93}$ Clinical studies have demonstrated that a single sub-anesthetic dose of the dissociative anesthetic ketamine induces rapid and sustained antidepressant actions in treatment-resistant patients. ${ }^{94}$ The focus of previous research has been to elucidate the molecular mechanisms underlying the unique antidepressant effects of ketamine, with a view to developing new drug therapies that mimic these effects without the associated adverse effects. ${ }^{95}$ Recent studies have shown that miRNAs may be involved in the antidepressant mechanism of action of ketamine. Yang et al determined the expression profile of miRNAs in the hippocampus of rats treated with ketamine. $^{96}$ The results suggested that multiple miRNAs were aberrantly expressed in the hippocampus of ketamine-injected rats (18 miRNAs were significantly down-regulated, while 22 miRNAs were significantly up-regulated). ${ }^{96}$ Among the down-regulated miRNAs, miR-206 has been reported to target the 3'UTR of BDNF mRNA and modulate the expression of BDNF. ${ }^{97}$ Taken together, the overexpression of miR-206 significantly attenuated ketamine-induced up-regulation of BDNF; ${ }^{96}$ these findings indicate that miRNA-206 may represent a novel therapeutic target relevant to the anti-depressive effects of ketamine.

ECT is a clinically effective method for alleviating the symptoms of refractory depression. ${ }^{98}$ Preclinical studies have found that levels of miR-212 were significantly increased in the rat dentate gyrus following both acute and chronic ECT. ${ }^{99}$ Moreover, miR-212 levels also increased in whole blood following chronic ECT, and were positively correlated with miR-212 levels in the dentate gyrus. ${ }^{99}$ These results suggest that alterations in miRNA expression may be informative in relation to the antidepressant mechanism of action of ECT. ${ }^{99}$ A clinical study compared healthy controls with patients diagnosed with major depression who were treatment-resistant in 
Table 2 miRNA Candidates Which are Correlated to the Antidepressant Treatment in Depression

\begin{tabular}{|c|c|c|c|c|c|c|c|c|c|}
\hline \multirow[t]{2}{*}{ MiRNA } & \multicolumn{4}{|l|}{ SSRIs } & \multicolumn{2}{|l|}{ Snris } & \multirow[t]{2}{*}{ Ketamine } & \multirow[t]{2}{*}{ Antidepressant } & \multirow[t]{2}{*}{ ECT } \\
\hline & Fluoxetine & Paroxetine & Escitalopram & Citalopram & Duloxetine & Desvenlafaxine & & & \\
\hline miR-let-7b & & $\uparrow[102]$ & & & & & & & \\
\hline miR- let-7c & & $\uparrow[102]$ & & & & & & & $\downarrow[67]$ \\
\hline miR-7d & & & $\uparrow[85]$ & & & & & & \\
\hline miR-7e & & & $\uparrow[85]$ & & & & & & \\
\hline miR-7g & & & $\uparrow[85]$ & & & & & & \\
\hline miR-7f & & & $\uparrow[85]$ & & & & & & \\
\hline miR-18a & & & & & $\uparrow[14]$ & & & & \\
\hline miR-20b-3p & & & & & & & & $\uparrow[30]$ & \\
\hline miR-22 & & & $\uparrow[85]$ & & & & & & \\
\hline miR-24-3p & & & $\downarrow[70]$ & & $\downarrow[70]$ & & & & \\
\hline miR-26a & & & $\uparrow[85]$ & & & & & & \\
\hline miR-26b & & & $\uparrow[85]$ & & & & & & \\
\hline miR-29b-2 & & & $\uparrow[85]$ & & & & & & \\
\hline miR-30a-5p & & $\uparrow[87]$ & & & & & & & \\
\hline miR-30b-3p & & $\downarrow[102]$ & & & & & & & \\
\hline miR-30d & & & $\uparrow[85]$ & & & & & & \\
\hline miR-34a-5p & & $\downarrow[2 I]$ & & & & & & & \\
\hline miR-34c-5p & & & $\downarrow[85]$ & & & & & & \\
\hline miR-103 & & & $\uparrow[85]$ & & & & & & \\
\hline miR-106a-5p & & & & & & & & & $\downarrow[101]$ \\
\hline miR-106b & & & $\uparrow[85]$ & & & & & & \\
\hline miR-124 & $\downarrow[19]$ & & & & & & & $\downarrow[3 \mid]$ & \\
\hline$m i R-124$ & & & & $\uparrow[72]$ & & & & & \\
\hline$m i R-124 a$ & & & & & $\downarrow[14]$ & & & & \\
\hline miR-126-3p & & & & & & & & & $\downarrow[101]$ \\
\hline$m i R-128$ & & & $\uparrow[85]$ & & & & & & \\
\hline miR-130b & & & $\uparrow[85]$ & & & & & & \\
\hline miR-132 & & & $\uparrow[85]$ & & & & & & \\
\hline miR-132 & & & & & $\uparrow[14]$ & & & & \\
\hline miR-132 & & $\uparrow[102]$ & & & & & & & \\
\hline miR-132 & & & & $\downarrow[72]$ & & & & & \\
\hline miR-133a & & & & & & & & $\uparrow[30]$ & \\
\hline miR-134 & & & & & $\downarrow[14]$ & & & & \\
\hline miR-135 & $\uparrow[17]$ & & & & & & & & \\
\hline miR-140-3p & & & $\uparrow[85]$ & & & & & & \\
\hline miR-145 & & & & & & & & $\uparrow[30]$ & \\
\hline miR-146a-5p & & & $\downarrow[70]$ & & $\downarrow[70]$ & & & & \\
\hline miR-146b-5p & & & $\downarrow[70]$ & & $\downarrow[70]$ & & & & \\
\hline miR-I5I-3p & & $\uparrow[88,102]$ & & & & & & & \\
\hline miR-183 & & & $\uparrow[85]$ & & & & & & \\
\hline miR-|9| & & & $\uparrow[85]$ & & & & & & \\
\hline miR-206 & & & & & & & $\downarrow[97]$ & & \\
\hline $\operatorname{miR}-212$ & & & & & & & & & $\uparrow[100]$ \\
\hline$m i R-22 I-3 p$ & & $\downarrow[2 I]$ & & & & & & & \\
\hline miR-320a & $\uparrow[8 \mid]$ & & & & & & & & \\
\hline miR-326 & & & $\downarrow[84]$ & & & & & & \\
\hline miR-33I-5p & & & & & & & & $\downarrow[30]$ & \\
\hline miR-335 & & & $\uparrow[85]$ & & & & & & \\
\hline miR-36I-5p & & & $\uparrow[85]$ & & & & & & \\
\hline $\mathrm{miR}-374 \mathrm{~b}$ & & & $\uparrow[85]$ & & & & & & \\
\hline miR-409-3p & & & & & & & & $\uparrow[30]$ & \\
\hline miR-4I0 & & & & & & & & $\uparrow[30]$ & \\
\hline miR-425-3p & & & & & $\downarrow[70]$ & & & & \\
\hline
\end{tabular}


Table 2 (Continued).

\begin{tabular}{|c|c|c|c|c|c|c|c|c|c|}
\hline \multirow[t]{2}{*}{ MiRNA } & \multicolumn{4}{|l|}{ SSRIs } & \multicolumn{2}{|l|}{ Snris } & \multirow[t]{2}{*}{ Ketamine } & \multirow[t]{2}{*}{ Antidepressant } & \multirow[t]{2}{*}{ ECT } \\
\hline & Fluoxetine & Paroxetine & Escitalopram & Citalopram & Duloxetine & Desvenlafaxine & & & \\
\hline $\begin{array}{l}\text { miR-433 } \\
\text { miR-45। } \\
\text { miR-45 la } \\
\text { miR-485-3p } \\
\text { miR-489 } \\
\text { miR-494 } \\
\text { miR-500 } \\
\text { miR-502-3p } \\
\text { miR-505 } \\
\text { miR-572 } \\
\text { miR-574-3p } \\
\text { miR-589 } \\
\text { miR-598-5p } \\
\text { miR-629 } \\
\text { miR-663a } \\
\text { miR-664 } \\
\text { miR-770-5p } \\
\text { miR-1202 } \\
\text { miR-197I } \\
\text { miR-3074-5p }\end{array}$ & $\begin{array}{l}\uparrow[51] \\
\uparrow[8 \mid] \\
\uparrow[8 \mid] \\
\uparrow[51] \\
\uparrow[8 \mid] \\
\downarrow[75]\end{array}$ & $\uparrow[21]$ & $\begin{array}{l} \\
\\
\uparrow[85] \\
\uparrow[85] \\
\uparrow[85] \\
\uparrow[85] \\
\uparrow[85] \\
\uparrow[85] \\
\uparrow[85] \\
\uparrow[85] \\
\downarrow[85]\end{array}$ & $\begin{array}{l}\uparrow[18] \\
\end{array}$ & ¿[70] & & & $\begin{array}{l}\uparrow[30] \\
\uparrow[30]\end{array}$ & \\
\hline
\end{tabular}

Notes: $\uparrow$ : upregulated, $\downarrow$ : downregulated; Antidepressant: according to the currently accepted therapeutic guidelines from the American Psychiatric Association.

order to identify peripheral miRNA biomarkers that could be used for diagnosis and prediction of response to ECT. ${ }^{100}$ It has been shown that the baseline expression of let-7c was $50 \%$ lower in treatment-resistant patients who received ECT. ${ }^{100}$ Another clinical study demonstrated that ECT treatment normalized the elevated blood levels of miR-126-3p and miR-106a-5p in patients with psychotic depression. ${ }^{101}$

Table 2 shows the miRNA candidate biomarkers for monitoring response to treatment with SSRIs, SNRIs, ketamine, and ECT in patients with depression. These results clearly suggest the potential role of miRNAs as putative biomarkers for assessing the therapeutic response in depression.

\section{Conclusions}

Depression has emerged as an important public health problem that often results in disability. The identification of objective biomarkers and improvement of diagnostic accuracy is critical for effective depression therapy. Although multiple lines of evidence suggest that miRNAs represent promising diagnostic and therapeutic biomarkers for depression, their clinical application remains limited.
Future Directions: Depression-related miRNA research must be more rigorous in design (for example, subjects with depression should be classified into responders and non-responders in order to more accurately assess miRNAs as indicators of treatment effect); the sample size should be enlarged in a homogeneous population; and repetition for the verification of results should be performed. Such directions would advance further exploration of the pathological mechanism of miRNAs in depression and assist in identifying objective markers that can effectively predict the efficacy of an antidepressant. Moreover, research on miRNAs in depression needs to be combined with research results in other fields, such as brain function imaging and gene polymorphism data, to develop personalized and targeted clinical treatment for depression.

\section{Funding}

This project was supported by Key Research and Development Programme of Anhui Province of China (201904a07020009).

\section{Disclosure}

The author reports no conflicts of interest in this work. 


\section{References}

1. World Health Organization. Depression. March 22, 2018. Available from: https://www.who.int/news-room/fact-sheets /detail/depression. Accessed November 30, 2019.

2. Beekman ATF, Spijker J. Personalised diagnosis and treatment of depression. Tijdschr Psychiatr. 2018;60(3):156-160.

3. Xu T, Fang Y, Rong A, et al. Flexible combination of multiple diagnostic biomarkers to improve diagnostic accuracy. BMC Med Res Methodol. 2015;15:94. doi:10.1186/s12874-015-0085-Z

4. Strawbridge R, Young AH, Cleare AJ. Biomarkers for depression: recent insights, current challenges and future prospects. Neuropsychiatr Dis Treat. 2017;13:1245-1262. doi:10.2147/ NDT.S114542

5. Bartel DP. MicroRNAs. Cell. 2004;116(2):0-297. doi:10.1016/ S0092-8674(04)00045-5

6. Cao DD, Lu L, Chan WY. MicroRNAs: key regulators in the central nervous system and their implication in neurological diseases. Int J Mol Sci. 2016;17(6):842. doi:10.3390/ijms17060842

7. Blandford SN, Galloway DA, Moore CS. The roles of extracellular vesicle microRNAs in the central nervous system. Glia. 2018;(12). doi:10.1002/glia.23445

8. Zhang Y, Zhang D, Wang F, et al. Serum miRNAs panel (miR-16$2^{*}$, miR-195, miR-2861, miR-497) as novel non-invasive biomarkers for detection of cervical cancer. Sci Rep. 2015;5:17942. doi:10.1038/srep17942

9. Sundarbose K, Kartha RV, Subramanian S. MicroRNAs as biomarkers in cancer. Diagnostics. 2013;3(1):84-104. doi:10.3390/ diagnostics3010084

10. Salehi M, Mohammadreza SPD. Exosomal miRNAs as novel cancer biomarkers: challenges and opportunities. J Cell Physiol. 2018;233(1):6370-6380. doi:10.1002/jcp.26481

11. Wang S, Wang JQ, Lv XW. Exosomal miRNAs as biomarkers in the diagnosis of liver disease. Biomark Med. 2017;11(6):491-501. doi:10.2217/bmm-2017-0011

12. Tavakolizadeh J, Roshanaei K, Salmaninejad A, et al. MicroRNAs and exosomes in depression: potential diagnostic biomarkers. J Cell Biochem. 2017;119(5):3783-3797.

13. Maffioletti E, Tardito D, Gennarelli M, et al. Micro spies from the brain to the periphery: new clues from studies on microRNAs in neuropsychiatric disorders. Front Cell Neurosci. 2014;8(8):75 doi:10.3389/fncel.2014.00075

14. Pan B, Liu Y. Effects of duloxetine on microRNA expression profile in frontal lobe and hippocampus in a mouse model of depression. Int J Clin Exp Pathol. 2015;8(11):15454-15461.

15. Mitchell PS, Parkin RK, Kroh EM, et al. Circulating microRNAs as stable blood-based markers for cancer detection. Proc Natl Acad Sci U S A. 2008;105(30):10513-10518. doi:10.1073/pnas.0804549105

16. Wang $X$, Sundquist $K$, Hedelius $A$, et al. Circulating microRNA-144-5p is associated with depressive disorders. Clin Epigenetics. 2015;7:69. doi:10.1186/s13148-015-0099-8

17. Issler O, Haramati S, Paul E, et al. MicroRNA 135 is essential for chronic stress resiliency, antidepressant efficacy, and intact serotonergic activity. Neuron. 2014;83(2):344-360. doi:10.1016/j. neuron.2014.05.042

18. Lopez JP, Lim R, Cruceanu C, et al. miR-1202 is a primate-specific and brain-enriched microRNA involved in major depression and antidepressant treatment. Nat Med. 2014;20(7):764-768. doi:10.1038/nm.3582

19. Dwivedi Y. microRNA-124: a putative therapeutic target and biomarker for major depression. Expert Opin Ther Targets. 2017;21(7):653-656. doi:10.1080/14728222.2017.1328501

20. Roy B, Dunbar M, Shelton RC, et al. Identification of MicroRNA-124-3p as a putative epigenetic signature of major depressive disorder. Neuropsychopharmacology. 2017;42(4):86 4-875. doi:10.1038/npp.2016.175
21. Kuang W-H, Dong Z-Q, Tian L-T, et al. MicroRNA-451a, microRNA-34a-5p, and microRNA-221-3p as predictors of response to antidepressant treatment. Braz J Med Biol Res. 2018;51(7):e7212. doi:10.1590/1414-431x20187212

22. Li Y-J, Xu M, Gao Z-H, et al. Alterations of serum levels of BDNF-related miRNAs in patients with depression. PLoS One. 2013;8(5):e63648. doi:10.1371/journal.pone.0063648

23. Camkurt MA, Acar Ş, Coşkun S, et al. Comparison of plasma MicroRNA levels in drug naive, first episode depressed patients and healthy controls. J Psychiatr Res. 2015;69:67-71. doi:10. 1016/j.jpsychires.2015.07.023

24. Sun N, Lei L, Wang Y, et al. Preliminary comparison of plasma notch-associated microRNA-34b and $-34 \mathrm{c}$ levels in drug naive, first episode depressed patients and healthy controls. J Affect Disord. 2016;194:109-114. doi:10.1016/j.jad.2016.01.017

25. Fan H-M, Sun X-Y, Niu W, et al. Altered microRNA expression in peripheral blood mononuclear cells from young patients with schizophrenia. J Mol Neurosci. 2015;56(3):562-571. doi:10.1007/ s12031-015-0503-Z

26. Fisar Z, Raboch J. Depression, antidepressants, and peripheral blood components. Neuro Endocrinol Lett. 2008;29(1):17-28.

27. Lai C-Y, Yu S-L, Hsieh MH, et al. MicroRNA expression aberration as potential peripheral blood biomarkers for schizophrenia. PLoS One. 2011;6(6):e21635. doi:10.1371/journal.pone.0021635

28. Gladkevich A, Kauffman HF, Korf J. Lymphocytes as a neural probe: potential for studying psychiatric disorders. Prog Neuropsychopharmacol Biol Psychiatry. 2004;28(3):559-576. doi:10.1016/j.pnpbp.2004.01.009

29. Liang Y, Ridzon D, Wong L, et al. Characterization of microRNA expression profiles in normal human tissues. BMC Genomics. 2007;8(1):166. doi:10.1186/1471-2164-8-166

30. Belzeaux R, Bergon A, Jeanjean V, et al. Responder and nonresponder patients exhibit different peripheral transcriptional signatures during major depressive episode. Transl Psychiatry. 2012;2:e185. doi:10.1038/tp.2012.112

31. He S, Liu X, Jiang K, et al. Alterations of microRNA-124 expression in peripheral blood mononuclear cells in pre- and post-treatment patients with major depressive disorder. J Psychiatr Res. 2016;78:65-71. doi:10.1016/j.jpsychires.20 16.03.015

32. Fan H-M, Sun X-Y, Guo W, et al. Differential expression of microRNA in peripheral blood mononuclear cells as specific biomarker for major depressive disorder patients. $J$ Psychiatr Res. 2014;59:45-52. doi:10.1016/j.jpsychires.2014.08.007

33. Wan Y, Liu Y, Wang $X$, et al. Identification of differential microRNAs in cerebrospinal fluid and serum of patients with major depressive disorder. PLoS One. 2015;10(3):e0121975. doi:10.1371/journal.pone. 0121975

34. Ajit SK. Circulating microRNAs as biomarkers, therapeutic targets, and signaling molecules. Sensors. 2012;12(3):3359-3369. doi: $10.3390 / \mathrm{s} 120303359$

35. Xu YY, Ge JF, Liang J, et al. Nesfatin-1 and cortisol: potential novel diagnostic biomarkers in moderate and severe depressive disorder. Psychol Res Behav Manag. 2018;11:495-502. doi:10.2147/PRBM.S183126

36. Garbett KA, Vereczkei A, Kálmán S, et al. Coordinated messenger RNA/microRNA changes in fibroblasts of patients with major depression. Biol Psychiatry. 2015;77(3):256-265. doi:10.1016/j. biopsych.2014.05.015

37. Kálmán S, Garbett KA, Vereczkei A, et al. Metabolic stress-induced microRNA and mRNA expression profiles of human fibroblasts. Exp Cell Res. 2014;320(2):343-353. doi:10.1016/j.yexcr.2013.10.019

38. Manier DH, Shelton RC, Ellis TC, et al. Human fibroblasts as a relevant model to study signal transduction in affective disorders. $J$ Affect Disord. 2000;61(1):51-58. 
39. Gibson SA, Korade Ž, Shelton RC, et al. Oxidative stress and glutathione response in tissue cultures from persons with major depression. J Psychiatr Res. 2012;46(10):1326-1332. doi:10.10 16/j.jpsychires.2012.06.008

40. Gallego JA, Gordon ML, Claycomb K, et al. In vivo microRNA detection and quantitation in cerebrospinal fluid. $J$ Mol Neurosci Mn. 2012;47(2):243-248. doi:10.1007/s12031-012-9731-7

41. Mckeever PM, Schneider R, Taghdiri F, et al. MicroRNA expression levels are altered in the cerebrospinal fluid of patients with young-onset Alzheimer's disease. Mol Neurobiol. 2018;55 (12):8826-8841. doi:10.1007/s12035-018-1032-x

42. Akers JC, Hua W, Li H, et al. A cerebrospinal fluid microRNA signature as biomarker for glioblastoma. Oncotarget. 2017;8 (40):68769-68779. doi:10.18632/oncotarget.v8i40

43. Song M-F, Dong J-Z, Wang Y-W, et al. CSF miR-16 is decreased in major depression patients and its neutralization in rats induces depression-like behaviors via a serotonin transmitter system. J Affect Disord. 2015;178:25-31. doi:10.1016/j. jad.2015.02.022

44. Campbell S, Macqueen G. The role of the hippocampus in the pathophysiology of major depression. J Psychiatry Neurosci Jpn. 2004;29(6):417-426.

45. Masi G, Brovedani P. The hippocampus, neurotrophic factors and depression. CNS Drugs. 2011;25(11):913-931. doi:10.2165/ 11595900-000000000-00000

46. Trost W, Frã $1 / 4$ Hholz S. The hippocampus is an integral part of the temporal limbic system during emotional processing: comment on "The quartet theory of human emotions: an integrative and neurofunctional model" by S. Koelsch et al. Phys Life Rev. 2015;13:87-88. doi:10.1016/j.plrev.2015.04.012

47. Mcgowan D. Neuroendocrinology: hormonal hippocampus. Nat Rev Neurosci. 2006;7(7):329. doi:10.1038/nrn1917

48. Kim JJ, Diamond DM. The stressed hippocampus, synaptic plasticity and lost memories. Nat Rev Neurosci. 2002;3(6):453-462. doi: $10.1038 / \mathrm{nrn} 849$

49. Sahay A, Hen R. Hippocampal neurogenesis and depression. Novartis Found Symp. 2008;289:152.

50. Yau Y, Lau BW-M, Tong J-B, et al. Hippocampal neurogenesis and dendritic plasticity support running-improved spatial learning and depression-like behaviour in stressed rats. PLoS One. 2011;6 (9):e24263. doi:10.1371/journal.pone.0024263

51. O'Connor RM, Grenham S, Dinan TG, et al. microRNAs as novel antidepressant targets: converging effects of ketamine and electroconvulsive shock therapy in the rat hippocampus. Int J Neuropsychopharmacol. 2013;16(8):1885-1892. doi:10.10 17/S1461145713000448

52. Bao AM, Meynen G, Swaab DF. The stress system in depression and neurodegeneration: focus on the human hypothalamus. Brain Res Rev. 2008;57(2):531-553. doi:10.1016/j.brainresrev.2007. 04.005

53. Yang J, Zhang L, Cao -L-L, et al. MicroRNA-99a is a potential target for regulating hypothalamic synaptic plasticity in the peri/ postmenopausal depression model. Cells. 2019;8(9):1081. doi:10. 3390/cells 8091081

54. Hastings RS, Parsey RV, Oquendo MA, et al. Volumetric analysis of the prefrontal cortex, amygdala, and hippocampus in major depression. Neuropsychopharmacology. 2004;29(5):952-959. doi:10.1038/sj.npp.1300371

55. Sibille E, Arango V, Galfalvy HC, et al. Gene expression profiling of depression and suicide in human prefrontal cortex. Neuropsychopharmacology. 2004;29(2):351-361. doi:10.1038/sj. npp. 1300335

56. Smalheiser NR, Lugli G, Rizavi HS, et al. MicroRNA expression is down-regulated and reorganized in prefrontal cortex of depressed suicide subjects. PLoS One. 2012;7(3):e33201. doi:10.1371/journal.pone.0033201
57. Serafini G, Pompili M, Hansen KF, et al. The involvement of MicroRNAs in major depression, suicidal behavior, and related disorders: a focus on miR-185 and miR-491-3p. Cell Mol Neurobiol. 2014;34(1):17-30. doi:10.1007/s10571-013-9997-5

58. Lopez JP, Fiori LM, Gross JA, et al. Regulatory role of miRNAs in polyamine gene expression in the prefrontal cortex of depressed suicide completers. Int $J$ Neuropsychopharmacol. 2014;17(1):23-32. doi:10.1017/S1461145713000941

59. Gorinski N, Bijata M, Prasad S, et al. Attenuated palmitoylation of serotonin receptor 5-HT1A affects receptor function and contributes to depression-like behaviors. Nat Commun. 2019;10 (1):3924. doi:10.1038/s41467-019-11876-5

60. Bai Y, Song L, Dai G, et al. Antidepressant effects of magnolol in a mouse model of depression induced by chronic corticosterone injection. Steroids. 2018;135:73-78.

61. Dwivedi Y, Roy B, Lugli G, et al. Chronic corticosterone-mediated dysregulation of microRNA network in prefrontal cortex of rats: relevance to depression pathophysiology. Transl Psychiatry. 2015;5:e682. doi:10.1038/tp.2015.175

62. Xu -Y-Y, Ge J-F, Qin G, et al. Acute, but not chronic, stress increased the plasma concentration and hypothalamic mRNA expression of NUCB2/nesfatin-1 in rats. Neuropeptides. 2015;54:47-53. doi:10.1016/j.npep.2015.08.003

63. Gu Z, Pan J, Chen L. MiR-124 suppression in the prefrontal cortex reduces depression-like behavior in mice. Biosci Rep. 2019;39(9). doi:10.1042/BSR20190186

64. Doan L, Manders T, Wang J. Neuroplasticity underlying the comorbidity of pain and depression. Neural Plast. 2015;2015:504691. doi:10.1155/2015/504691

65. Satyanarayanan SK, Shih Y-H, Wen Y-R, et al. miR-200a-3p modulates gene expression in comorbid pain and depression: molecular implication for central sensitization. Brain Behav Immun. 2019;82:230-238. doi:10.1016/j.bbi.2019.08.190

66. Cao M-Q, Chen D-H, Zhang C-H, et al. Screening of specific microRNA in hippocampus of depression model rats and intervention effect of Chaihu Shugan San. Zhongguo Zhong Yao Za Zhi. 2013;38(10):1585-1589.

67. Gheysarzadeh A, Sadeghifard N, Afraidooni L, et al. Serumbased microRNA biomarkers for major depression: miR-16, miR-135a, and miR-1202. J Res Med Sci. 2018;23:69. doi:10.4103/jrms.JRMS_879_17

68. Bai M, Zhu X, Zhang Y, et al. Abnormal hippocampal BDNF and miR-16 expression is associated with depression-like behaviors induced by stress during early life. PLoS One. 2012;7(10): e46921. doi:10.1371/journal.pone.0046921

69. Chin-Chuen Lin C-TL, Sun M-H, Huang T-L. Increased levels of miR-30e, miR-132, miR-185, and miR212 at baseline and increased brain-derived neurotrophic factor protein and mRNA levels after treatment in patients with major depressive disorder. Neuropsychiatry. 2017;7(6):920-926.

70. Liang HB, He J-R, Tu X-Q, et al. MicroRNA-140-5p: a novel circulating biomarker for early warning of late-onset post-stroke depression. J Psychiatr Res. 2019;115:129-141. doi:10.1016/j. jpsychires.2019.05.018

71. Torres-Berrío A, Lopez JP, Bagot RC, et al. DCC confers susceptibility to depression-like behaviors in humans and mice and is regulated by miR-218. Biol Psychiatry. 2016;81(4):306. doi:10.1016/j.biopsych.2016.08.017

72. Schmidt U, Herrmann L, Hagl K, et al. Therapeutic action of fluoxetine is associated with a reduction in prefrontal cortical miR-1971 expression levels in a mouse model of posttraumatic stress disorder. Front Psychiatry. 2013;4:66. doi:10.3389/fpsyt.2013.00066

73. Schmidt HD, Shelton RC, Duman RS. Functional biomarkers of depression: diagnosis, treatment, and pathophysiology. Neuropsychopharmacology. 2011;36(12):2375-2394. doi:10.1038/ npp.2011.151 
74. Belzeaux R, Lin R, Turecki G. Potential use of MicroRNA for monitoring therapeutic response to antidepressants. CNS Drugs. 2017;31(4):253-262. doi:10.1007/s40263-017-0418-Z

75. Cully M. Mood disorders: microRNA in depression and treatment response. Nat Rev Drug Discovery. 2014;13(8):576.

76. Gitlin M. Selective Serotonin Reuptake Inhibitors (SSRIs). Encycl Stress. 2007;44(3):440-443.

77. Baudry A, Mouillet-Richard S, Schneider B, et al. miR-16 targets the serotonin transporter: a new facet for adaptive responses to antidepressants. Science. 2010;329(5998):1537-1541. doi:10.1126/ science. 1193692

78. Vasu MM, Anitha A, Takahashi T, et al. Fluoxetine increases the expression of miR-572 and miR-663a in human neuroblastoma cell lines. PLoS One. 2016;11(10):e0164425. doi:10.1371/journal. pone.0164425

79. Fang Y, Qiu Q, Zhang S, et al. Changes in miRNA-132 and miR-124 levels in non-treated and citalopram-treated patients with depression. J Affect Disord. 2016;6:745-751. doi:10.1016/j. jad.2017.11.090

80. Emslie GJ, Ventura D, Korotzer A, et al. Escitalopram in the treatment of adolescent depression: a randomized placebo-controlled multisite trial. J Am Acad Child Adolesc Psychiatry. 2009;48 (7):721-729. doi:10.1097/CHI.0b013e3181a2b304

81. Zhong H, Haddjeri N, Sánchez C. Escitalopram, an antidepressant with an allosteric effect at the serotonin transporter-a review of current understanding of its mechanism of action. Psychopharmacology. 2012;219(1):1-13. doi:10.1007/s00213011-2463-5

82. Zhang Y, Wang Y, Wang L, et al. Dopamine receptor D2 and associated microRNAs are involved in stress susceptibility and resistance to escitalopram treatment. Int $J$ Neuropsychopharmacol. 2015;18(8):pyv025-pyv025. doi:10.1093/ijnp/pyv025

83. Bocchio-Chiavetto L, Maffioletti E, Bettinsoli P, et al. Blood microRNA changes in depressed patients during antidepressant treatment. Eur Neuropsychopharmacol. 2013;23(7):602-611. doi:10.1016/j.euroneuro.2012.06.013

84. Mertens C, Pintens H. Paroxetine in the treatment of depression. A double-blind multicenter study versus mianserin. Acta Psychiatr Scand. 2010;77(6):683-688. doi:10.1111/j.16000447.1988.tb05188.x

85. Angelucci F, Croce N, Spalletta G, et al. Paroxetine rapidly modulates the expression of brain-derived neurotrophic factor mrna and protein in a human glioblastoma-astrocytoma cell line. Pharmacology. 2011;87(1-2):5-10. doi:10.1159/000322528

86. Oved K, Morag A, Pasmanik-Chor M, et al. Genome-wide miRNA expression profiling of human lymphoblastoid cell lines identifies tentative SSRI antidepressant response biomarkers. Pharmacogenomics. 2012;13(10):1129-1139. doi:10.2217/pgs. 12.93

87. Goldstein D, Lu Y, Detke MJ, et al. Duloxetine in the treatment of depression: a double-blind placebo-controlled comparison with paroxetine. Eur Psychiatry. 2002;12(4):214.
88. Schueler YB, Koesters M, Wieseler B, et al. A systematic review of duloxetine and venlafaxine in major depression, including unpublished data. Acta Psychiatr Scand. 2015;123(4):247-265. doi:10.1111/acps.2011.123.issue-4

89. Montgomery SA. Tolerability of serotonin norepinephrine reuptake inhibitor antidepressants. CNS Spectr. 2008;13(S11):27-33. doi:10.1017/S1092852900028297

90. Monteleone F, Caputo M, Felice Tecce M, et al. Duloxetine in the treatment of depression: an overview. Cent Nerv Syst Agents Med Chem. 2011;11(3):174-183. doi:10.2174/187152411798047807

91. Lopez JP, Fiori LM, Cruceanu C, et al. MicroRNAs $146 \mathrm{a} / \mathrm{b}-5$ and 425-3p and 24-3p are markers of antidepressant response and regulate MAPK/Wnt-system genes. Nat Commun. 2017;8:15497. doi:10.1038/ncomms 15497

92. Lopez JP, Pereira F, Richard-Devantoy S, et al. Co-variation of peripheral levels of miR-1202 and brain activity and connectivity during antidepressant treatment. Neuropsychopharmacology. 2017;42(10):2043-2051. doi:10.1038/npp.2017.9

93. Berman RM, Cappiello A, Anand A, et al. Antidepressant effects of ketamine in depressed patients. Biol Psychiatry. 2000;47 (4):351-354. doi:10.1016/S0006-3223(99)00230-9

94. Zanos P, Gould TD. Mechanisms of ketamine action as an antidepressant. Mol Psychiatry. 2018;23(4):801-811. doi:10.10 38/mp.2017.255

95. Dewilde KE, Levitch CF, Murrough JW, et al. The promise of ketamine for treatment-resistant depression: current evidence and future directions. Ann N Y Acad Sci. 2015;1345(1):47-58. doi:10.1111/nyas. 12646

96. Yang X, Yang Q, Wang X, et al. MicroRNA expression profile and functional analysis reveal that miR-206 is a critical novel gene for the expression of BDNF induced by ketamine. Neuromolecular Med. 2014;16(3):594-605. doi:10.1007/s12017014-8312-z

97. Soon-Tae Lee MD, Chu K, Jung K-H, et al. miR-206 regulates brain-derived neurotrophic factor in Alzheimer disease model. Ann Neurol. 2012;72(2):269-277. doi:10.1002/ana.v72.2

98. Read J, Arnold C. Is electroconvulsive therapy for depression more effective than placebo? A systematic review of studies since 2009. Ethical Human Psychol Psychiatry. 2017;19 (1):5-23. doi:10.1891/1559-4343.19.1.5

99. Ryan KM, O’Donovan SM, Mcloughlin DM. Electroconvulsive stimulation alters levels of BDNF-associated microRNAs. Neurosci Lett. 2013;549(33):125-129. doi:10.1016/j.neulet.2013.05.035

100. Gururajan A, Naughton ME, Scott KA, et al. MicroRNAs as biomarkers for major depression: a role for let-7b and let-7c. Transl Psychiatry. 2016;6(8):e862. doi:10.1038/tp.2016.131

101. Kolshus E, Ryan KM, Blackshields G, Smyth P, Sheils O, McLoughlin DM. Peripheral blood microRNA and VEGFA mRNA changes following electroconvulsive therapy: implications for psychotic depression. Acta Psychiatr Scand. 2017;136 (6):594-606. doi:10.1111/acps.12821

Neuropsychiatric Disease and Treatment

Dovepress

\section{Publish your work in this journal}

Neuropsychiatric Disease and Treatment is an international, peerreviewed journal of clinical therapeutics and pharmacology focusing on concise rapid reporting of clinical or pre-clinical studies on a range of neuropsychiatric and neurological disorders. This journal is indexed on PubMed Central, the 'PsycINFO' database and CAS, an is the official journal of The International Neuropsychiatric Association (INA). The manuscript management system is completely online and includes a very quick and fair peer-review system, which is all easy to use. Visit http://www.dovepress.com/testimonials.php to read real quotes from published authors. 